\title{
Colonic Chicken Skin Mucosa is an Independent Endoscopic Predictor of Advanced Colorectal Adenoma
}

\author{
Eun Ju Chung ${ }^{1 *}$, Ji Young Lee ${ }^{1 *}$, Jaewon Choe ${ }^{1}$, Hye-Sook Chang $^{1}$, Jongcheol Kim ${ }^{1}$, Dong Hoon Yang ${ }^{2}$, \\ Byong Duk Ye ${ }^{2}$, Jeong-Sik Byeon ${ }^{2}$, Kyung-Jo Kim², Suk-Kyun Yang ${ }^{2}$, Jin-Ho Kim², Seung-Jae Myung ${ }^{2}$ \\ Health Screening and Promotion Center', Department of Gastroenterology², Asan Medical Center, University of Ulsan College of Medicine, \\ Seoul, Korea
}

Background/Aims: Chicken skin mucosa (CSM), surrounding colorectal adenoma, is an endoscopic finding with pale yellowspeckled mucosa; however, its clinical significance is unknown. This study aimed to evaluate the prevalence and clinical characteristics of CSM, and the association between colorectal carcinogenesis and CSM. Methods: This cross-sectional study was performed in 733 consecutive patients who underwent endoscopic polypectomy for colorectal adenoma after the screening of colonoscopy at the Asan Health Promotion Center between June 2009 and December 2011. The colonoscopic and pathological findings of colorectal adenoma including number, size, location, dysplasia, morphology, and clinical parameters were reviewed. Results: The prevalence of CSM was 30.7\% (225 of 733 patients), and most CSM-related adenomas were located in the distal colon (93.3\%). Histological analysis revealed lipid-laden macrophages in the lamina propria of the mucosa. Multivariate analyses showed that CSM was significantly associated with advanced pathology, including villous adenoma and high-grade dysplasia (odds ratio $[\mathrm{OR}], 2.078 ; 95 \%$ confidence interval $[\mathrm{CI}], 1.191-3.627 ; P=0.010$ ), multiple adenomas (i.e., $\geq 2$ adenomas; $\mathrm{OR}, 1.692 ; 95 \% \mathrm{CI}, 1.143-2.507 ; P=0.009$ ), and a protruding morphology (OR, 1.493; 95\% CI, 1.027-2.170; $P=0.036$ ). There were no significant differences in polyp size or clinical parameters between patients with and without CSM. Conclusions: CSMrelated adenoma was mainly found in the distal colon, and was associated with advanced pathology and multiple adenomas. CSM could be a potential predictive marker of the carcinogenetic progression of distally located colorectal adenomas.

(Intest Res 2015;13:318-325)

Key Words: Chicken skin mucosa; Colorectal adenoma; Carcinogenesis; Colonoscopy

\section{INTRODUCTION}

Mucosal abnormalities and molecular changes associated with colorectal adenomas have been reported since colonos-

Received July 3, 2014. Revised July 3, 2015. Accepted July 4, 2015. Correspondence to Seung-Jae Myung, Department of Gastroenterology, Asan Medical Center, University of Ulsan College of Medicine, 88 Olympic-ro 43-gil, Songpa-gu, Seoul 05505, Korea. Tel: +82-2-3010-3917, Fax: +82-2476-0824,E-mail: sjmyung@amc.seoul.kr

${ }^{*}$ Both authors contributed equally to this work.

Financial support: This research was supported by the Bio \& Medical Technology Development Program of the National Research Foundation (NRF), funded by the Korean Government (MEST) (No. 2011-0019632) and the Asan Institute for Life Sciences (No. 2013-261 and No. 2013-559). Conflict of interest: None. copy began to be widely used for the screening of colorectal cancer. ${ }^{1-3}$ However, the clinical significance of colonic mucosal abnormalities on endoscopy is still unknown. Among the endoscopic mucosal abnormalities in the colon, chicken skin mucosa (CSM) was first identified in 1998 and was described with specific morphologic changes surrounding a colorectal adenoma. ${ }^{4} \mathrm{CSM}$ is a pale yellow-speckled pattern of colonic mucosa on endoscopy; lipid-filled macrophages in the lamina propria are noted on histopathology. Previous findings might have suggested presence of colonic xanthoma in $\mathrm{CSM}^{1,3,5-7}$ but CSM demonstrates distinct features that only occur adjacent to colorectal neoplasms. ${ }^{4}$

Previous studies suggested that CSM was caused by colonic intestinal metaplasia, toxic factors from damaged in-

\footnotetext{
(c) Copyright 2015. Korean Association for the Study of Intestinal Diseases. All rights reserved.

This is an Open Access article distributed under the terms of the Creative Commons Attribution Non-Commercial License (http://creativecommons.org/licenses/by-nc/4.0)

which permits unrestricted non-commercial use, distribution, and reproduction in any medium, provided the original work is properly cited.
} 
traluminal mucosa, or a sign of previous mild damage. ${ }^{4,8,9}$ In children, CSM was associated with juvenile polyps, which is not a precancerous condition, and immunostaining for Ki-67 and p53 was not increased. ${ }^{10,11}$ CSM-positive juvenile polyps were larger than CSM-negative juvenile polyps in children, probably because of local mucosal damage rather than preneoplastic lesions. ${ }^{10}$ However, a recent study showed that colorectal neoplasm with CSM was associated with increased risk of carcinogenesis. ${ }^{12}$ Colorectal adenomas with CSM in adults demonstrated higher Ki-67 and COX-2 expression as markers of cell proliferation and lower caspase-3 expression as a marker of apoptosis compared to colorectal adenoma without CSM. ${ }^{12}$ In addition, the prevalence of CSM was higher in carcinoma patients (64.3\%) than in adenoma patients (22.1\%). Therefore, it is still unclear whether CSM is associated with carcinogenetic process or is only a traumarelated mucosal change.

We evaluated the prevalence and clinical characteristics of patients with CSM, and whether CSM is a useful predictor of advanced colorectal adenoma. We also assessed the association between the existence of CSM and other risk factors for colorectal adenoma.

\section{METHODS}

\section{Subjects}

This cross-sectional study was conducted using a prospective registry of health check-up participants who underwent endoscopic mucosal resection (EMR) of colonic adenomas at the Asan Health Screening and Promotion Center between June 2009 and December 2011 following a screening colonoscopy. Patients who received a forceps biopsy of small polyps to avoid pathologic misdiagnosis and those with a prior history of colorectal cancer or IBD were excluded. All screened patients received details of the screening program in written, and a standard questionnaire related to their personal medical history, present medications, family history, and lifestyle. Physical examinations, laboratory assays, imaging studies, and endoscopies were performed after fasting for $>12$ hours on the day of examination. We retrospectively reviewed these laboratory findings and questionnaires, which was approved by the Asan Medical Center institutional review board.

\section{Colonoscopy and EMR}

The bowel was prepared using $4 \mathrm{~L}$ of polyethylene glycol solution. All examinations were performed by experienced colonoscopists using high-definition colonoscopy (CF$\mathrm{H}_{60 \mathrm{AI}}{ }^{\circledR}$; Olympus, Tokyo, Japan). During colonoscopy, the location, the number, and the size of polyps (estimated using the opened biopsy forceps) were recorded, and photos were taken of $\geq 10$ colon landmarks (terminal ileum, ileocecal valve, cecum, ascending colon, hepatic flexure, transverse colon, splenic flexure, descending colon, sigmoid colon, and rectum). When a polyp was found, pictures were taken at close range and the distant view during endoscopy to identify the characteristics of polyps. EMR was performed in cases of a polyp size $>5 \mathrm{~mm}$ or unresected adenoma during screening colonoscopy. All resected polyps were retrieved and reviewed by expert gastrointestinal pathologists, according to the Vienna classification. ${ }^{13}$ The diagnosis of CSM was determined by reviewing the colonoscopy images. Two blinded expert colonoscopists reviewed the colonoscopic findings by accessing the characteristics of the patients and the pathologic reports of polyps. Inter-observer variation was measured by Kappa statistic and the value was $0.629 .{ }^{14}$ For the patients with multiple colorectal adenomas associated with CSM, the most advanced lesion was used for the classification of the subjects.

\section{Definition of Terms Used in This Study}

The locations of colonic polyps were divided into the proximal colon (cecum, ascending colon, hepatic flexure, and transverse colon) and the distal colon (splenic flexure, descending colon, sigmoid colon, and rectum). Advanced pathology meant a colonic adenoma with $>25 \%$ villous structure, high-grade dysplasia, or carcinoma in situ.

\section{Statistical Analysis}

The relationship between the presence of CSM, clinical factors, and the characteristics of the colonic adenomas was analyzed using the $\chi^{2}$ test, whereas continuous variables were examined using the Student $t$-test. Logistic regression model was used to investigate the relationship between the advanced adenoma and the existence of CSM. Two-tailed $P$-values $<0.05$ was considered statistically significant. The Statistical Package for the Social Sciences version 21.0 (SPSS Inc.,Chicago, IL, USA) was used for all analyses. 


\section{RESULTS}

\section{Baseline Characteristics of the Study Population}

A total of 753 patients were eligible for inclusion. Of these, 20 patients were excluded in the final analysis because of a prior history of colorectal cancer $(\mathrm{n}=3)$ or an incomplete questionnaire $(\mathrm{n}=17)$. The mean age of the enrolled 733 patients was $56.5 \pm 8.5$ years; 563 patients $(76.8 \%)$ were men (Table 1). The mean number of adenomas per patient was $2.6 \pm 2.0$, with a median size of $9 \mathrm{~mm}$ (range $=4-30 \mathrm{~mm}$ ). The number of adenomas was represented as the sum of found polyps during initial colonoscopy and polypectomy. Among endoscopically resected adenomas, $90.5 \%$ were tubular adenomas ( $\mathrm{n}=663), 4.9 \%$ were villous adenomas $(\mathrm{n}=36)$, and $4.6 \%$ were high-grade dysplasia $(\mathrm{n}=24)$ (Table 2$)$.

\section{Clinical Characteristics of the Patients With CSM- Surrounding Adenoma}

Of all 733 patients, 225 (30.7\%) had CSM surrounding the adenoma in the colon. CSM adjacent to colorectal adenomas demonstrated tiny yellow speckles around adenoma (Fig. 1).
H\&E CSM demonstrated foamy macrophages in the lamina propria (Fig. 2). Patients with CSM were younger than those without CSM (54.9 \pm 8.7 years vs. $57.1 \pm 8.3$ years, respectively; $P=0.001)$. However, there were no significant differences in terms of BMI, smoking status, alcohol consumption, family history of colorectal cancer, metabolic syndrome, or the levels of total cholesterol, fasting glucose, triglyceride, or insulin.

\section{Association Between Characteristics of Adenoma and Existence of CSM}

The prevalence of high-grade dysplasia and carcinoma in situ was significantly higher in adenoma with CSM than without CSM ( $7.1 \%$ vs. $3.5 \%$, respectively; $P=0.003$ ). The advance pathology (e.g., villous adenoma, high-grade dysplasia, or carcinoma) was significantly associated with the presence of CSM (15.1\% in the adenomas with CSM vs. $7.1 \%$ without CSM; $P=0.001$ ). The presence of CSM was associated with adenoma multiplicity. The mean number of adenomas was $2.9 \pm 2.3$ in patients with CSM and $2.5 \pm 1.9$ in patients without CSM $(P=0.017)$. The prevalence of multiple adenomas $(\geq 2$ adenomas per patient) was also higher in patients with CSM than in patients without CSM $(P=0.004)$. CSM-related ad-

Table 1. Baseline Characteristics of Study Population

\begin{tabular}{|c|c|c|c|c|}
\hline Variable & $\begin{array}{c}\text { Total } \\
(n=733)\end{array}$ & $\begin{array}{l}\text { Absence of CSM } \\
(n=508)\end{array}$ & $\begin{array}{l}\text { Presence of CSM } \\
(n=225)\end{array}$ & $P$-value \\
\hline Age & $56.5 \pm 8.5$ & $57.1 \pm 8.3$ & $54.9 \pm 8.7$ & 0.001 \\
\hline Sex (Male:Female) & $563: 170$ & $392(77.2)$ & $171(76.0)$ & 0.776 \\
\hline $\mathrm{BMI}\left(\mathrm{kg} / \mathrm{m}^{2}\right)$ & $24.9 \pm 2.9$ & $24.9 \pm 2.9$ & $24.8 \pm 3.1$ & 0.738 \\
\hline Systolic blood pressure $(\mathrm{mmHg})$ & $123.8 \pm 13.7$ & $124.1 \pm 13.7$ & $122.9 \pm 13.6$ & 0.276 \\
\hline Diastolic blood pressure (mmHg) & $78.8 \pm 9.9$ & $79.1 \pm 9.9$ & $78.3 \pm 9.8$ & 0.336 \\
\hline Waist circumference $(\mathrm{cm})$ & $87.3 \pm 8.5$ & $87.3 \pm 8.4$ & $87.1 \pm 8.9$ & 0.744 \\
\hline Smoking & $474(64.7)$ & $333(65.6)$ & $141(62.7)$ & 0.451 \\
\hline Consumption of alcohol & $339(46.2)$ & $237(46.7)$ & $102(45.3)$ & 0.741 \\
\hline Family history of colorectal cancer & $40(5.5)$ & $28(5.5)$ & $12(5.3)$ & 0.922 \\
\hline Metabolic syndrome & $287(39.2)$ & $169(33.3)$ & $84(37.3)$ & 0.501 \\
\hline Total cholesterol (mg/dL) & $198.8 \pm 37.6$ & $198.8 \pm 36.6$ & $198.5 \pm 40.1$ & 0.907 \\
\hline Triglyceride (mg/dL) & $132.7 \pm 70.9$ & $131.0 \pm 68.5$ & $136.2 \pm 76.2$ & 0.365 \\
\hline LDL cholesterol (mg/dL) & $124.7 \pm 33.3$ & $124.4 \pm 31.9$ & $125.4 \pm 36.1$ & 0.685 \\
\hline HDL cholesterol (mg/dL) & $52.8 \pm 13.4$ & $53.4 \pm 13.7$ & $51.4 \pm 12.6$ & 0.056 \\
\hline Glucose (mg/dL) & $106.5 \pm 21.6$ & $107.5 \pm 23.5$ & $104.2 \pm 16.2$ & 0.055 \\
\hline Insulin ( $\mu \mid \mathrm{U} / \mathrm{mL})$ & $8.3 \pm 6.0$ & $8.4 \pm 6.2$ & $7.9 \pm 5.3$ & 0.313 \\
\hline
\end{tabular}

Values are presented as mean \pm SD or $n(\%)$.

CSM, chicken skin mucosa. 
Table 2. Characteristics of Adenoma Related to Chicken Skin Mucosa (CSM)

\begin{tabular}{|c|c|c|c|c|}
\hline Variable & Total $(n=733)$ & $\begin{array}{l}\text { Absence of CSM } \\
(n=508)\end{array}$ & $\begin{array}{l}\text { Presence of CSM } \\
(n=225)\end{array}$ & $P$-value \\
\hline Mean no. of adenoma & $2.6 \pm 2.0$ & $2.5 \pm 1.9$ & $2.9 \pm 2.3$ & 0.017 \\
\hline Single adenoma & $231(31.5)$ & $177(34.8)$ & $54(24.0)$ & \\
\hline Multiple adenomas $(\geq 2)$ & $502(68.5)$ & $331(65.2)$ & $171(76.0)$ & \\
\hline Location of polyps & & & & $<0.001$ \\
\hline Distal colon only & $186(25.4)$ & $90(17.7)$ & $96(42.7)$ & \\
\hline Proximal colon only & $254(34.7)$ & $239(47.0)$ & $15(6.7)$ & \\
\hline Both & $293(40.0)$ & $179(35.2)$ & $114(50.7)$ & \\
\hline High-grade dysplasia* & $24(4.6)$ & $18(3.5)$ & $16(7.1)$ & \\
\hline Morphology & & & & 0.007 \\
\hline Flat (Is, Ila, Ilb, LST) & $251(34.2)$ & $190(37.4)$ & $61(27.1)$ & \\
\hline Protruding (Ip, Isp) & $482(65.8)$ & $318(62.6)$ & $164(72.9)$ & \\
\hline
\end{tabular}

Values are presented as mean \pm SD or $n(\%)$.

*High-grade dysplasia included carcinoma in situ.

LST, laterally spreading tumor.
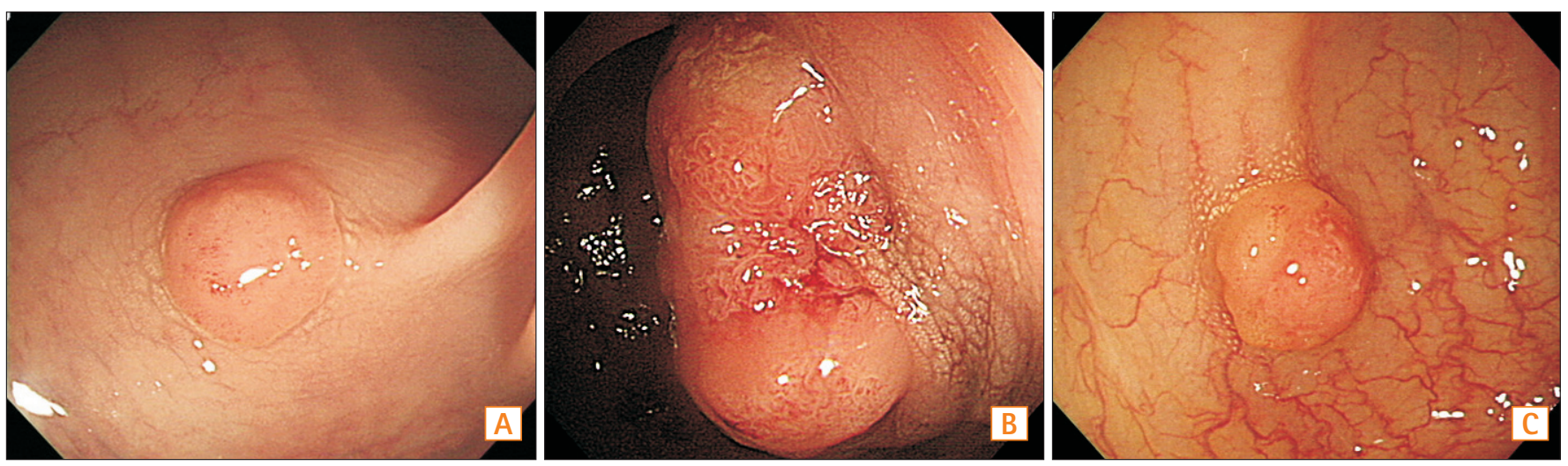

Fig. 1. Endoscopic features of chicken skin mucosa. (A) Endoscopic features of a chicken skin mucosa (CSM), including tiny yellow speckles adjacent to a tubular adenoma. (B) Endoscopic features of a CSM, including dense yellow speckles adjacent to a colonic adenocarcinoma. (C) Endoscopic features of a CSM, including yellow speckles adjacent to early colonic adenocarcinoma.

enomas were frequently observed in the distal colon (93.3\%) and in protruding type adenomas (72.9\%). However, the median adenoma size was not associated with the presence of CSM (Table 2).

\section{Multivariable Analysis of Association Between Adenoma and CSM}

In univariate logistic regression, CSM-related adenomas were associated with younger age (OR, 0.969; 95\% CI, 0.951-0.988; $P=0.001$ ), multiple adenomas (OR, 1.693; 95\% CI, 1.186-2.418; $P=0.004$ ), protruding morphology (OR, 

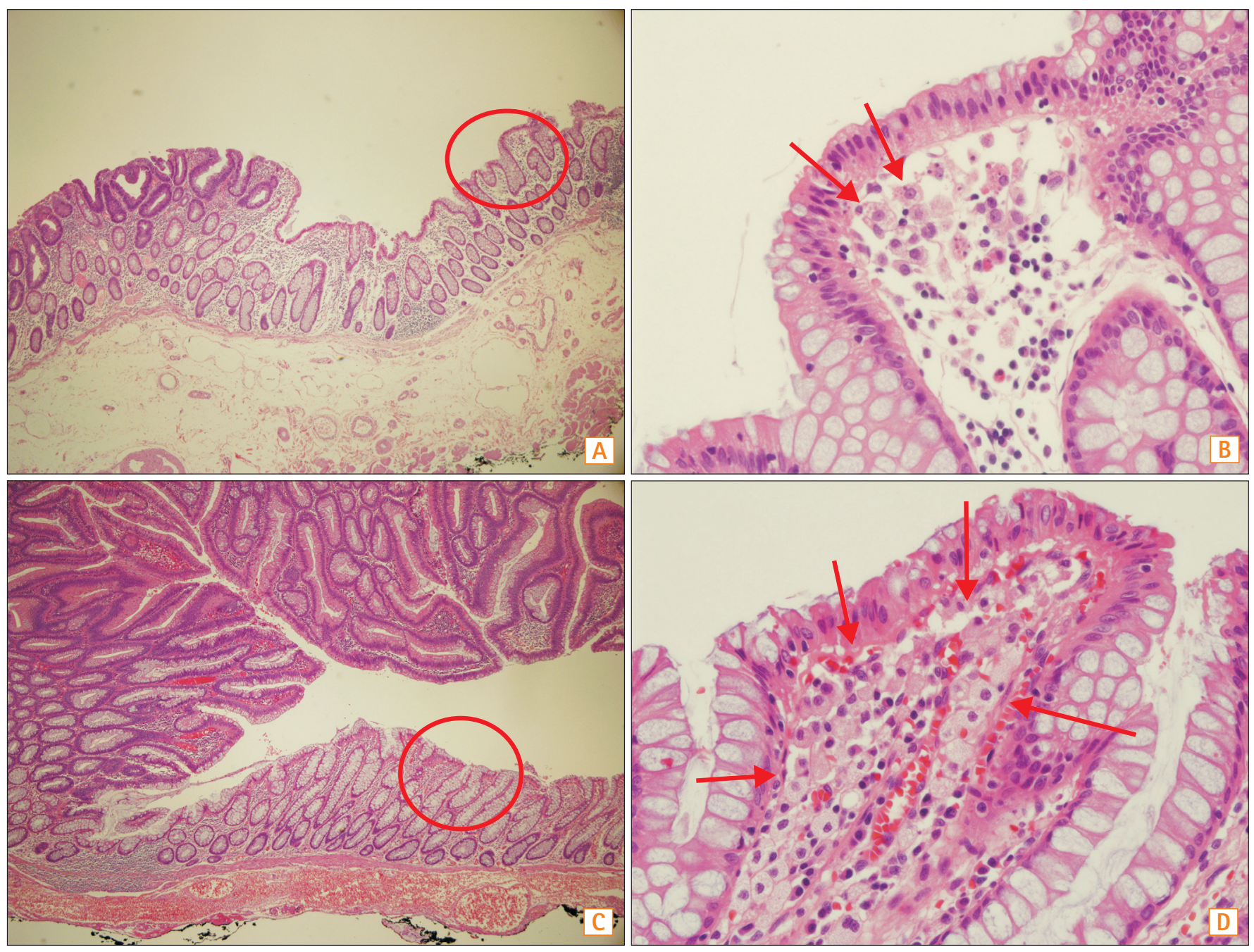

Fig. 2. Histologic appearances of chicken skin mucosa. (A) Histological appearance of tubular adenoma and the surrounding mucosa (HEtE, $\times 40)$. (B) Histological appearance of chicken skin mucosa (CSM) surrounding a tubular adenoma, including foamy macrophages (arrow) located in the lamina propria ( $\mathrm{HEEE}, \times 400)$. (C) Histological appearance of colonic adenocarcinoma and the surrounding mucosa ( $\mathrm{HEEE}, \times 40)$. (D) Histological appearance of CSM surrounding a colonic adenocarcinoma, including densely deposited foamy macrophages (arrow) located in the lamina propria (HEEE, $\times 400)$.

1.606; 95\% CI, 1.138-2.267; $P=0.007)$, and advanced pathology (OR, 2.334; 95\% CI, 1.419-3.840; $P=0.001)$. In multivariate logistic regression, CSM-related adenoma was associated with younger age (OR, 0.960; 95\% CI, 0.937-0.983; $P=0.001)$, multiple adenomas (OR, 1.692; 95\% CI, 1.143-2.507; $P=0.009$ ), protruding morphology (OR, 1.493; 95\% CI, $1.027-2.170 ; P=0.036)$, and advanced pathology (OR, 2.078; 95\% CI, 1.191-3.627; $P=0.010$ ) (Table 3).

\section{Distribution of Lipid-Laden Macrophage Infiltration According to Pathology}

CSM is an aggregation of a large number of lipid-laden macrophages in the colorectal mucosa. Although we mostly use endoscopic images to detect CSM, we examined the distribution of lipid-laden macrophages in several cases after EMR. CSM with adenocarcinoma or high-grade dysplasia exhibited higher infiltration of lipid-laden macrophages in the lamina propria than CSM with tubular adenoma (Fig. 2). These findings are considerably equivalent with macroscopic view of CSM (Fig. 1).

\section{DISCUSSION}

CSM frequently surrounds polyps in the distal colon and rectum; however, its clinical prevalence remains unknown owing to inadequate research in this area. Our data showed that $30.7 \%$ of patients with colorectal adenoma or carcinoma 
Table 3. Logistic Regression Model of Characteristics of Adenoma and Chicken Skin Mucosa

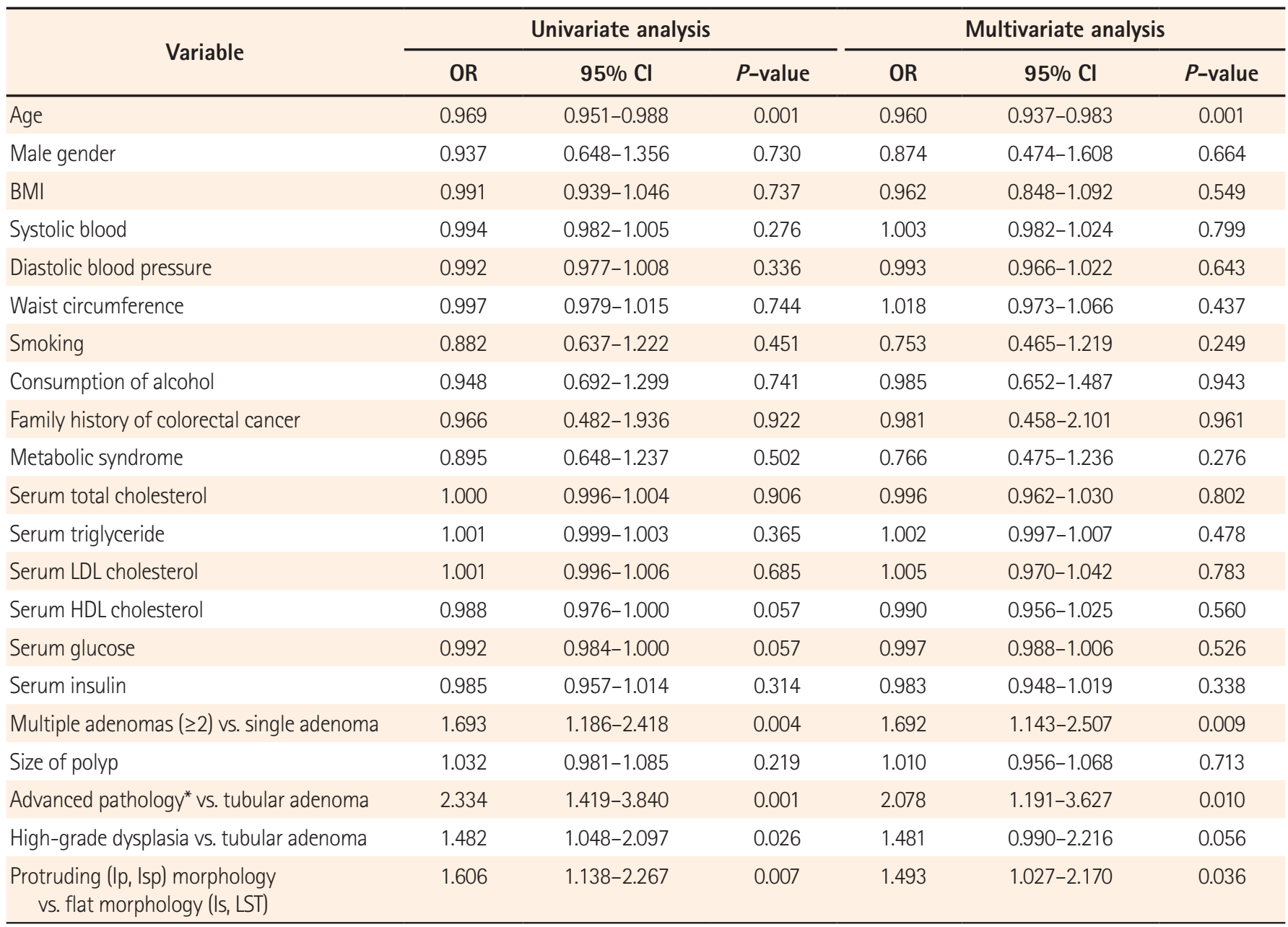

* Advanced pathology included villous adenoma, high-grade dysplasia, and carcinoma in situ.

LST, laterally spreading tumor.

demonstrated CSM. Advanced pathology (i.e. villous adenoma, dysplasia, and carcinoma) and multiplicity of adenoma were found to be associated with CSM. Patients with CSM also demonstrated a higher prevalence of synchronized polyps in both the distal and proximal colon compared to patients without CSM (50.6\% vs. $35.2 \%, P<0.001)$. Synchronous adenomas are high risk group for the development of advanced adenomas and cancer. ${ }^{15,16}$ The presence of distal polyps was helpful to predict advanced proximal neoplasia in asymptomatic old patients, and more careful examination is required in patients with distal polyps with $\mathrm{CSM}^{17}$

Our previous study revealed that a Western-style diet promotes colon carcinogenesis by recruiting macrophages in a mouse model. ${ }^{18}$ The risk factors for colorectal cancer include visceral obesity, metabolic syndrome, age $>60$ years, advanced pathology, and IBD. ${ }^{19-21}$ As per our current study, the presence of CSM is not associated with obesity, old age, metabolic syndrome, or other risk factors, and may be an independent endoscopic entity.

CSM with adenocarcinoma or high-grade dysplasia exhibited much higher infiltration of lipid-laden macrophages than CSM with tubular adenoma in the lamina propria (Fig. 2). Macrophages are key players in the inflammatory response, and the connection between chronic inflammation and tumorigenesis is well established. The number of mucosal macrophages appears to be correlated with the relative bacterial load, and their numbers decrease in the intestine of germ-free mice. ${ }^{22}$ The inflammatory microenvironment is important for cancer development, and tumor-associated macrophages (TAM) exert pro-tumor functions in this microenvironment. ${ }^{23}$ TAM promotes the growth, angiogenesis, and metastasis of various cancers by releasing growth fac- 
tors, cytokines, chemokines, and enzymes. The endoscopic features of CSM surrounding adenoma coincide with the infiltration of lipid-laden macrophages. It is unclear whether the macrophages are TAM, because macrophages in CSM reside in normal mucosa surrounding tumor; however, TAM are found in the stroma of tumors. ${ }^{24}$ Therefore, it is supposed that lipid-laden macrophages move towards the tumor and infiltrate the area around it. This phenomenon may express the extent of inflammatory activity and carcinogenetic progression of the tumor. Most adenomas with CSM in our present study were located in the sigmoid colon or rectum. Distal colon and rectum might have more chance of bowel inflammation by retaining stool, bacteria, and macrophages. A recent study reported that high numbers of macrophage population located in the spleen as an extra-medullary site. ${ }^{25}$ These findings support why CSM is mostly found in the distal colon, even though macrophages are widely distributed.

Previous data indicated that adenocarcinomas and colorectal adenomas with CSM expressed similar levels of Ki-67 and COX-2 proteins; survivin and caspase-3, key regulators of apoptosis, were suppressed in colorectal adenomas with CSM. ${ }^{12}$ Although these findings have important implications that the occurrence of colorectal cancer is associated with apoptosis, the sample size of the study was small and the clinical characteristics of the CSM patients were poorly characterized. It is uncertain whether CSM surrounding adult colorectal adenoma is a risk factor for colon carcinogenesis. CSM with juvenile polyps in children is suggested as the result of local mucosal trauma indicating that it is not a precursor to dysplasia, ${ }^{26}$ although CSM-positive polyps are larger than CSM-negative polyps. However, this finding is debatable because juvenile polyps are hamartomatous and post surveillance was not performed.

Our current study has limitations considering that small and minute-sized polyps were not analyzed. In addition, we could not test all samples of CSM around polyps owing to the retrospective nature of the study. Further biological experiments and prospective studies are needed to determine if the CSM is indeed a predictive marker of colorectal cancer development. Nevertheless, the current study is, to our knowledge, the first to report the prevalence and clinical characteristics of colorectal neoplasms with CSM found in the screening field. Detection of advanced neoplasia is very important to determine the interval of surveillance colonoscopy ${ }^{27}$ and CSM may provide important information for post-polypectomy surveillance.

In conclusion, the present retrospective analyses showed that CSM was associated with tumor multiplicity and ad- vanced histology in distally located colon adenomas. Obesity, metabolic syndrome, and old age were not associated with CSM. It is uncertain whether CSM surrounding colorectal adenoma is a risk factor for colon carcinogenesis; however, CSM is a distinctive marker of advanced pathology of colorectal adenoma. It can provide valuable insights for future research in this area and for planning post-polypectomy surveillance.

\section{REFERENCES}

1. Nakasono M, Hirokawa M, Muguruma N, et al. Colorectal xanthomas with polypoid lesion: report of 25 cases. APMIS 2004;112:3-10.

2. Okano A, Takakuwa H, Nakamura T, Ohana M, Kusumi F, Nabeshima M. Rectosigmoid xanthoma as a predictive marker for sporadic rectosigmoid cancer. J Clin Gastroenterol 2011;45:837838.

3. Miliauskas JR. Rectosigmoid (colonic) xanthoma: a report of four cases and review of the literature. Pathology 2002;34:144147.

4. Shatz BA, Weinstock LB, Thyssen EP, Mujeeb I, DeSchryver K. Colonic chicken skin mucosa: an endoscopic and histological abnormality adjacent to colonic neoplasms. Am J Gastroenterol 1998;93:623-627.

5. Weinstock LB, Shatz BA, Saltman RJ, Deschryver K. Xanthoma of the colon. Gastrointest Endosc 2002;55:410.

6. Moran AM, Fogt F. 70-year-old female presenting with rectosigmoid (colonic) xanthoma and multiple benign polyps - case report. Pol J Pathol 2010;61:42-45.

7. Delgado Fontaneda E, Basagoiti ML, Aperribay A, Ruiz Rebollo L, Moretó Canela M. Xanthoma of the colon. Rev Esp Enferm Dig 1992;82:203-204.

8. Remmele W, Beck K, Kaiserling E. Multiple lipid islands of the colonic mucosa. A light and electron microscopic study. Pathol Res Pract 1988;183:336-346.

9. Bejarano PA, Aranda-Michel J, Fenoglio-Preiser C. Histochemical and immunohistochemical characterization of foamy histiocytes (muciphages and xanthelasma) of the rectum. Am J Surg Pathol 2000;24:1009-1015.

10. Nowicki MJ, Bishop PR, Subramony C, Wyatt-Ashmead J, May W, Crawford M. Colonic chicken-skin mucosa in children with polyps is not a preneoplastic lesion. J Pediatr Gastroenterol Nutr 2005;41:600-606.

11. El-Hodhod MA, Soliman AA, Hamdy AM, Abdel-Rahim AA, Abdel-Hamid FK. Fate and ultra-structural features of chicken skin mucosa around juvenile polyps. Acta Gastroenterol Belg 2011;74:17-21. 
12. Guan J, Zhao R, Zhang X, et al. Chicken skin mucosa surrounding adult colorectal adenomas is a risk factor for carcinogenesis. Am J Clin Oncol 2012;35:527-532.

13. Schlemper RJ, Riddell RH, Kato Y, et al. The Vienna classification of gastrointestinal epithelial neoplasia. Gut 2000;47:251255.

14. Viera AJ, Garrett JM. Understanding interobserver agreement: the kappa statistic. Fam Med 2005;37:360-363.

15. Lee KH, Kim HC, Yu CS, Myung SJ, Yang SG, Kim JC. Colonoscopic surveillance after curative resection for colorectal cancer with synchronous adenoma. Korean J Gastroenterol 2005;46:381-387.

16. Moon CM, Cheon JH, Choi EH, et al. Advanced synchronous adenoma but not simple adenoma predicts the future development of metachronous neoplasia in patients with resected colorectal cancer. J Clin Gastroenterol 2010;44:495-501.

17. Keum B, Yoon TJ, Choi JH, et al. Clinical value of distal colon polyps for prediction of advanced proximal neoplasia: the KASID prospective multicenter study. Intest Res 2005;3:121-126.

18. Kim IW, Myung SJ, Do MY, et al. Western-style diets induce macrophage infiltration and contribute to colitis-associated carcinogenesis. J Gastroenterol Hepatol 2010;25:1785-1794.

19. Cowey SL, Quast M, Belalcazar LM, et al. Abdominal obesity, insulin resistance, and colon carcinogenesis are increased in mutant mice lacking gastrin gene expression. Cancer 2005;103:2643-2653.

20. Aleksandrova K, Boeing H, Jenab M, et al. Metabolic syndrome and risks of colon and rectal cancer: the European prospective investigation into cancer and nutrition study. Cancer Prev Res (Phila) 2011;4:1873-1883.
21. Lukas M. Inflammatory bowel disease as a risk factor for colorectal cancer. Dig Dis 2010;28:619-624.

22. Niess JH, Adler G. Enteric flora expands gut lamina propria $\mathrm{CX}_{3} \mathrm{CR}^{+}$dendritic cells supporting inflammatory immune responses under normal and inflammatory conditions. J Immunol 2010;184:2026-2037.

23. Laoui D, Van Overmeire E, De Baetselier P, Van Ginderachter JA, Raes G. Functional relationship between tumor-associated macrophages and macrophage colony-stimulating factor as contributors to cancer progression. Front Immunol doi: 10.3389/fimmu.2014.00489. Published online 7 October 2014

24. Vendramini-Costa DB, Carvalho JE. Molecular link mechanisms between inflammation and cancer. Curr Pharm Des 2012;18:3831-3852.

25. Cortez-Retamozo V, Etzrodt M, Newton A, et al. Origins of tumor-associated macrophages and neutrophils. Proc Natl Acad Sci U S A 2012;109:2491-2496.

26. Nowicki MJ, Subramony C, Bishop PR, Parker PH. Colonic chicken skin mucosa: association with juvenile polyps in children. Am J Gastroenterol 2001;96:788-792.

27. Hong SP, Kim TI, Kim HG, et al. Clinical practice of surveillance colonoscopy according to the classification of colorectal intraepithelial neoplasia in Korea: high-grade dysplasia/ carcinoma in situ versus intramucosal carcinoma. Intest Res 2013;11:276-282. 